\section{An Android Application for Estimating Muscle Onset Latency using Surface EMG Signal}

\author{
Karimpour M..$^{1,2}$, Parsaei H. ${ }^{3,4}$, Rojhani-Shirazi Z. ${ }^{5}$, Sharifian \\ R. ${ }^{1 *}$, Yazdani F. ${ }^{5}$
}

\begin{abstract}
Background: Electromyography (EMG) signal processing and Muscle Onset Latency (MOL) are widely used in rehabilitation sciences and nerve conduction studies. The majority of existing software packages provided for estimating MOL via analyzing EMG signal are computerized, desktop based and not portable; therefore, experiments and signal analyzes using them should be completed locally. Moreover, a desktop or laptop is required to complete experiments using these packages, which costs.
\end{abstract}

Objective: Develop a non-expensive and portable Android application (app) for estimating MOL via analyzing surface EMG.

Material and Methods: A multi-layer architecture model was designed for implementing the MOL estimation app. Several Android-based algorithms for analyzing a recorded EMG signal and estimating MOL was implemented. A graphical user interface (GUI) that simplifies analyzing a given EMG signal using the presented app was developed too.

Results: Evaluation results of the developed app using 10 EMG signals showed promising performance; the MOL values estimated using the presented app are statistically equal to those estimated using a commercial Windows-based surface EMG analysis software (MegaWin 3.0). For the majority of cases relative error $<10 \%$. MOL values estimated by these two systems are linearly related, the correlation coefficient value $\sim 0.93$. These evaluations revealed that the presented app performed as well as MegaWin 3.0 software in estimating MOL.

Conclusion: Recent advances in smart portable devices such as mobile phones have shown the great capability of facilitating and decreasing the cost of analyzing biomedical signals, particularly in academic environments. Here, we developed an Android app for estimating MOL via analyzing the surface EMG signal. Performance is promising to use the app for teaching or research purposes.

\section{Keywords}

Electromyography, Surface EMG signal analysis, Muscle Onset Latency, Muscle Onset Latency Estimation, Android application

\section{Introduction}

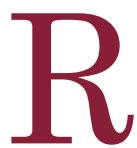
ecent advancements in compact, portable computing devices such as smartphones and tablets have created an opportunity for developing portable and inexpensive systems for analyzing medical data [1]. Portable medical devices improved the overall quality of medical services [2]. New technology such as telemedicine and eHealth allow experienced physicians to provide online services to sev-

${ }^{1}$ School of Management \& Medical Information

Sciences, Health Human

Resources Research

Center, Shiraz University

of Medical Sciences,

Shiraz, Iran

${ }^{2}$ Neuroscience Research

Center, Iran University

of Medical Sciences,

Tehran, Iran

${ }^{3}$ Department of Medical

Physics and Engineering

School of Medicine, Shi-

raz University of Medical

Sciences, Shiraz, Iran

${ }^{4}$ Shiraz Neuroscience

Research Center, Shiraz

University of Medical Sci-

ences, Shiraz, Iran

${ }^{5}$ Department of Physi-

otherapy, School of

Rehabilitation Sciences,

Shiraz University of

Medical Sciences, Shi-

raz, Iran

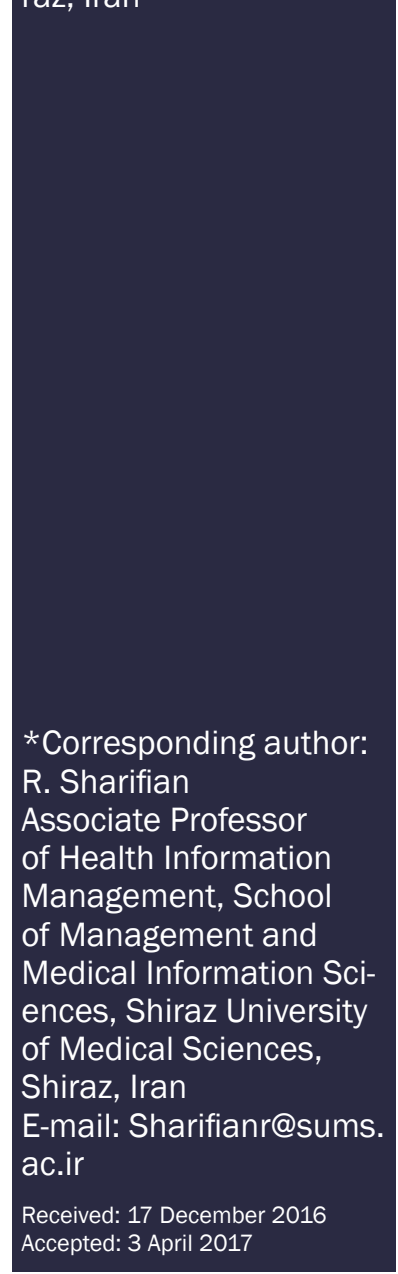


eral health care centers from a distance using telecommunications and information technology.

A subset of eHealth is mobile health (mHealth) that includes providing health information and services via mobile phone technology and wireless devices. The mHealth technology also includes employing basic tools of mobile phones such as Voice and Short Messaging Services, as well as more sophisticated features and applications including General Packet Radio Service (GPRS), 3G and 4G systems, Global Positioning System (GPS), and Bluetooth Technology to evaluate, diagnose or treat patients at distance [3]. One of the applications of mHealth technology is for physiological monitoring which includes reading, storing, processing and interpreting the physiologic parameters [4]. Up to now, several mHealth systems have been developed for acquiring and processing physiological signals especially for ECG signals [5-7].

An electromyographic (EMG) signal is the electrical activity of a contracting muscle. This electrical activity is generated by the active muscle cells / fibers of the contracting muscle. The EMG signals detected from a muscle using either surface electrode or needle electrode provide important information for physiological investigation and clinical examinations [8-11]. Muscle Onset Latency (MOL) [12] is the time interval between an electrical stimulus and the onset of mechanical response of the muscle in motor activity. This parameter reflects the conduction along the fastest fibers and is a useful index in the detection of carpal tunnel syndrome, neuropathy, diabetic neuropathy, neuromuscular junction disorders, motor neuron disease, nerve compression syndrome, the effect of diabetes on the nerves $[13,14]$. MOL can be measured using surface EMG. However, manual analysis of EMG signals cannot be completed in all circumstances. Manually analyzing EMG signals is costly, time-consuming, and may lead to errors when not carried out by an expert. To overcome these issues, several computer-based EMG analysis systems have been developed [11, 12, 15-21]. However, the majority of these software packages are desktop based, not portable and need a computer to run that may cost, so they might not be used widely in academic environments for teaching or research. Recent advances in smart portable devices such as mobile phones have shown the great capability of facilitating and decreasing the cost of analyzing biomedical signals, particularly in academic environments. Consequently, in this work, we investigated the possibility of developing an Android application (app) for estimating MOL via analyzing surface EMG signal. Detailed descriptions of the algorithm are provided in the following sections.

\section{Material and Methods}

The multi-layer architecture model shown in Figure 1 was designed for implementing the MOL estimation Android app. The developed multi-layer structure includes the following parts:

- Core layer: that manages through logical layer software, user interface, and database software layers.

- Data input layer: that performs an input data of middleware management through the middleware input file and input of electromyography device.

- Pre-processing algorithms layer: that includes pre-processing algorithms.

- Main processing algorithms layer: that contains the main processing algorithms, including estimation of muscle onset latency.

- Cloud layer: that includes cloud management and external database software.

In the developed software, MOL was estimated by thresholding approach in which the first point where the signal is greater than a threshold is considered as the EMG onset point. We followed the method presented in [22]. A vector of root mean square (RMS) values each of which was estimated using 15 data point samples of the signal is created. The 


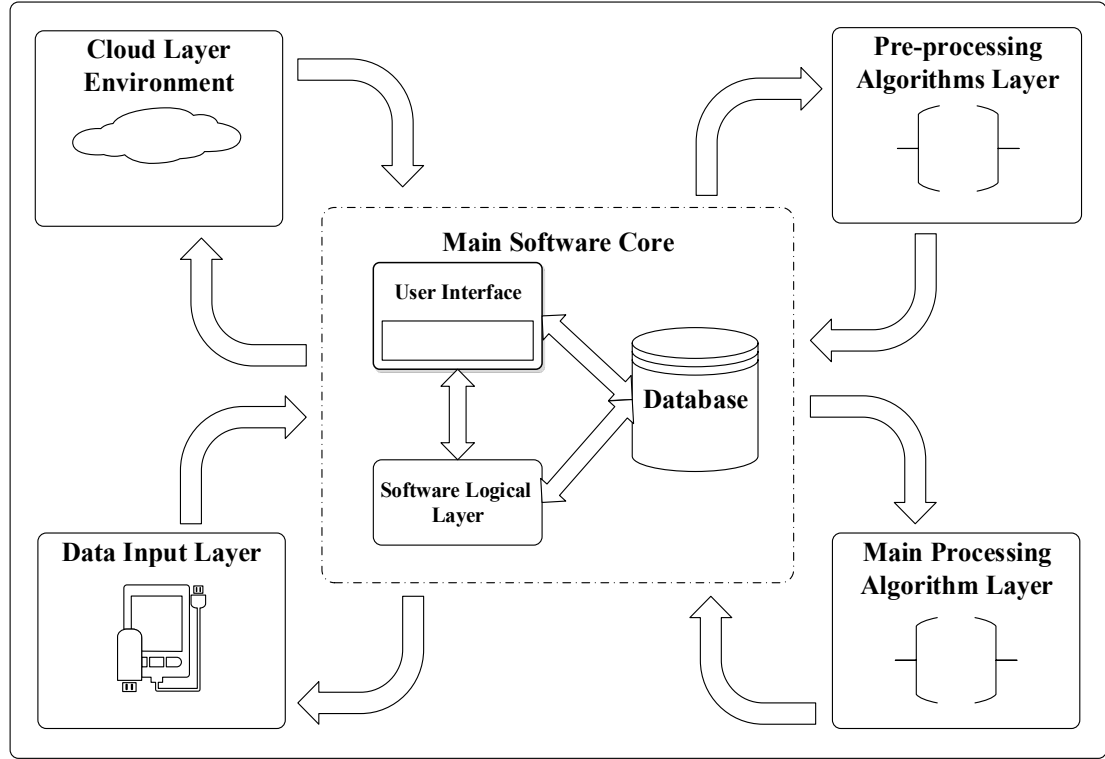

Figure 1: Multi-layer architecture model used in developing Muscle Onset Latency estimating application.

threshold value is set as a percentage $(25 \%)$ of the mean RMS values. The mean RMS is the sample mean of the calculated RMS values.

MOL estimation app was implemented by Android Development Tools (ADT) which is a plugin for the Eclipse IDE. Pre-processing and main processing algorithms (MOL estimation algorithm) were implemented as a separate middleware for each algorithm. The management of middleware layer was performed by preprocessing layer and main processing algorithm layer. Middleware of preprocessing and main processing algorithms were displayed after the installation process on Android OS in the list of software algorithms in the analysis of EMG signal. The internal structure of the database software was implemented by SQLite software and SQL. To connect the software to the software programming library, we used SQLite which is a part of Android programming from other libraries in the Eclipse tool. The external database software was implemented by cloud share-point environment list. In order to connect the software with share-point structure, we used the components of share-point integrator software programming. External database management software was done by the cloud layer and could be installed as a middleware on the Android operating system (OS) in the software to analyze surface EMG signals. Both Java and Android programming library standards were used in developing the app.

A key factor in implementing an algorithm on the Android OS is memory management [23]. In this work, for implementing the preprocessing algorithms and MOL estimation algorithm, the memory is divided into two parts: main memory and stick memory. In this way, only that part of the data which requires a processor with the Android OS is loaded in the main memory and the rest of the data will be loaded in the stick memory card. This method of memory management was used in compatible pre-processing electromyography algorithms and run on the Android OS.

\section{Results}

Figure 2 shows the environment of the developed MOL estimation App. In this figure, the estimated MOL for a given signal is shown. As shown, the app is user-friendly. The user 


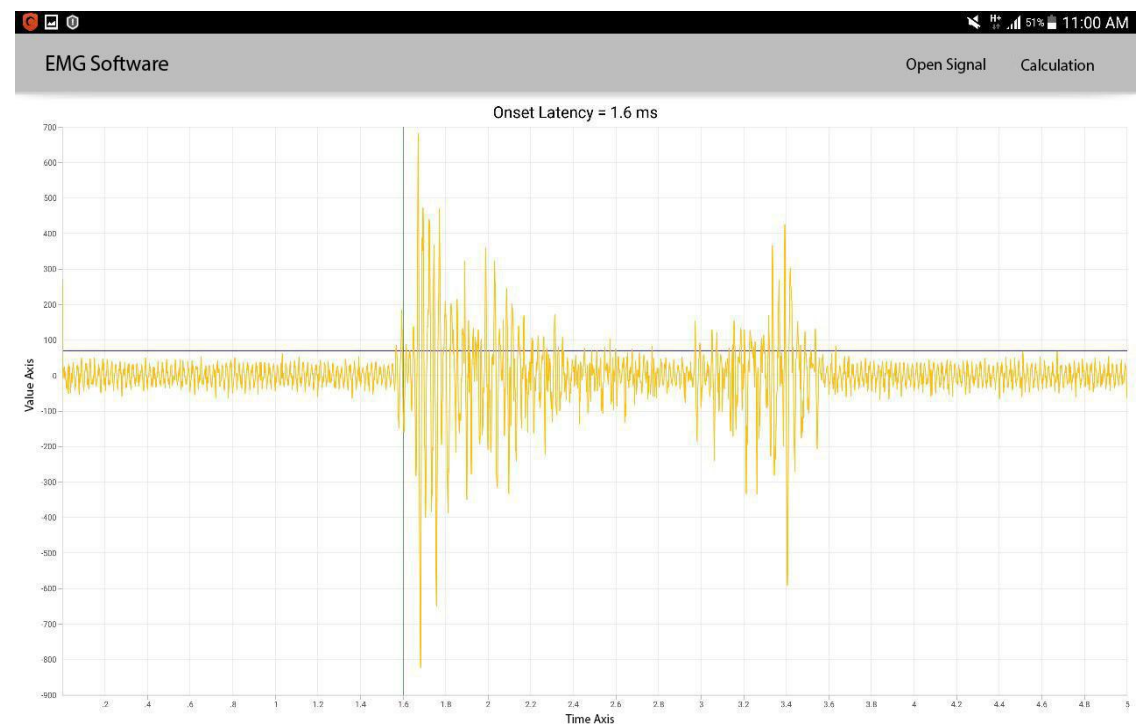

Figure 2: The user interface of the developed muscle onset latency estimation application. The estimated muscle onset latency value along with the position of the muscle actitivity onset point on the EMG signal (vertical line) and the employed threshold value (horizontal line).

can load the signal using "Open Signal” icon and then push "Calculation" icon to analyze the signal estimate MOL.

The performance of the developed app in estimating MOL was evaluated using 10 surface EMG signals from 10 healthy subjects. The signals were acquired using Digital Telemet- ric ME6000 EMG System from biceps muscle of the participant's dominant upper extremity during active elbow flexion movement. The MOL value for each case was estimated using MegaWin 3.0 Software and considered here as "gold standard". The results are summarized in Table 1. Nonparametric (Mann-Whitney)

Table 1: Comparison of Muscle Onset Latency values estimated using the developed App and MegaWin 3.0.

\begin{tabular}{ccccc}
$\begin{array}{c}\text { EMG Signal } \\
\text { Index }\end{array}$ & $\begin{array}{c}\text { MegaWin } \\
\mathbf{3 . 0}(\mathbf{m s})\end{array}$ & $\begin{array}{c}\text { Android } \\
\text { App (ms) }\end{array}$ & $\begin{array}{c}\text { Absolute } \\
\text { Error (ms) }\end{array}$ & $\begin{array}{c}\text { Relative } \\
\text { Error (\%) }\end{array}$ \\
\hline 1 & 2.0 & 2.2 & -0.2 & -10.0 \\
\hline 2 & 1.5 & 1.4 & 0.1 & 6.7 \\
\hline 3 & 1.5 & 1.6 & -0.1 & -6.7 \\
\hline 4 & 0.8 & 1.0 & -0.2 & -25.0 \\
\hline 5 & 1.6 & 1.4 & 0.2 & 12.5 \\
\hline 6 & 1.7 & 1.8 & -0.1 & -5.9 \\
\hline 7 & 1.9 & 2.0 & -0.1 & -5.3 \\
\hline 8 & 1.9 & 2.1 & -0.2 & -10.5 \\
\hline 9 & 1.9 & 1.8 & 0.1 & 5.3 \\
\hline 10 & 2.3 & 2.2 & 0.1 & 4.3 \\
\hline Mean & 1.71 & 1.75 & & \\
\hline STD & 0.40 & 0.39 & &
\end{tabular}


was used to compare the MOL values estimated using the developed app and gold standard values.

\section{Discussion}

Henriques et al. [24] discussed the importance of data collection in the medical industry. According to this study, traditional methods of data collection such as pen and paper are costly and time-consuming. Recent advancements in smart portable devices such as mobile phones have shown the great capability of facilitating and decreasing the cost of recording and analyzing medical data. In this work, we developed an Android app for analyzing EMG signal to estimate MOL. Yunju et al. discussed that age and gender are two fundamental factors in examining muscle onset latency [25]; thus, these two factors along with the name and surname of the patient are recorded in the database of the app. In order to understand the internal structure of the database software and how these structures connected, entity relationship diagram is used. Entity relationship diagram helps the programmer to understand the relationship between data structures and implement the database. Saving information to create a database is an important advantage of the derived app because such database can be used for further analysis or applications such as developing knowledge-based decision systems.

A software or an application, in fact, is organized instructions that manage system components to do a special task [26]. Here, multi-layered software design for implementing an EMG software analysis is presented. This multi-layered architecture makes the designed software flexible [27]. The developed software is divided into a central core and four layers. The central core supports a three-layer architecture model. The task of the core layer is managing the internal layers and other four layers of EMG analysis software. Each of the layers manages the installed middleware on the Android OS to communicate with their core software.

An external database software model was designed to share data with EMG. Based on the results, this model has two parts: the core database with the cloud environment and the cloud database structure. Communication core which inserts, edits, deletes and displays operations of the data was designed as a middleware which has the opportunity to work with different cloud environments. The data stored in the cloud need to be protected, and providing a safe and efficient sharing of data on the cloud through mobile phones needs to have encryption algorithms [28]. In order to maintain data security in the cloud environment, we considered an encryption module in core communication with a cloud environment. The duty of this module is to encrypt and decrypt the encrypted stored data. We need to implement the software and use the cloud technology because we can create a field in which the data can be interpreted elsewhere by the software by professionals and users of the software, and through data sharing, the data can be sent to the intended expert by the cloud environment for analyzing and estimating the muscle onset latency. By the use of the cloud environment, the problem of the need for permanent presence of medical professionals in the health centers will be solved and the professionals are given the accessibility to read medical data, from medical devices, out of health care centers. Using the cloud environment to share the electromyography data could be the perfect solution for efficient access to electromyography data for the experts.

There are several reasons why we chose Android OS for developing this MOL estimation app. It is an open source operating system; programmers have access to the Android source code easily and can freely write their own programs. It was estimated that around $79.0 \%$ of all smartphones and around $62.6 \%$ of the tablets sold between April and June 2013 had Android OS [29]. We need to implement the mentioned plan on the Android OS 
because by considering the capabilities of the Android open source, its components can be used in other applications for the mentioned project development. In addition, due to the increasing use of Android OS in smart portable devices such as mobile phones and tablets, it offers the ability to the mentioned plan to implement and use this software on the mobile phones or tablets of most users.

Finally, a key factor in using an app for medical application is its accuracy. Evaluation results of the developed app using 10 EMG signals showed that the developed app can estimate MOL well. Comparing with a commercial EMG analyzes software developed under Windows OS (Table 1), we found that there was no significant difference between the MOL values estimated using our app and those estimated using the employed desktopbased software. As shown in this table, for the majority of the signals relative error is $<10 \%$, that confirms that for the most of the cases the values provided by MegaWin 3.0 software and the portable system presented here are close. The correlations between MOL values estimated by the developed App and those estimated by MegaWin 3.0 software are shown in Figure 3. As shown, MOL values estimated

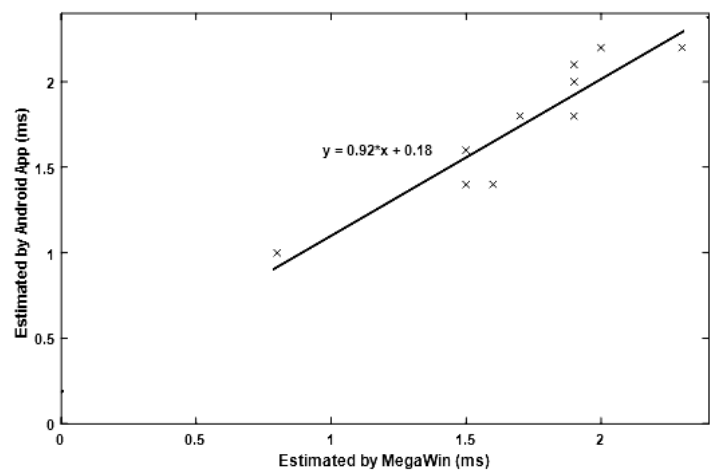

Figure 3: Correlation between $\mathrm{MOL}$ values estimated by the developed App and those estimated by MegaWin 3.0 software. These values provided by the App were significantly correlated with those estimated using software. by these two systems are linearly related, the correlation coefficient value is about 0.93 . All these analyses show that the derived App performed well in estimating MOL value. Nevertheless, the derived App should be evaluated using a large data set to accept its accuracy and reliability and validity.

\section{Conclusion}

Recent advances in smart portable devices such as mobile phones have shown the great capability of facilitating and decreasing the cost of analyzing biomedical signals, particularly in academic environments. Nowadays, mobile health applications are widely used for education, data collection, remote monitoring services, emergency medical services, and other health services. In this work, we investigated the possibility of developing an Android app for analyzing EMG signal to estimate MOL. Cloud environment technology embedded in this system for data sharing, provided this facility to rehabilitation professionals to access and analyze EMG signals outside the health center. In addition, this characteristic assists the physician to provide service and professional advice to different centers. In short, the accuracy of the developed app in estimating MOL along with its advantages over existing systems is promising; however, before using this app in a laboratory, it should be evaluated using a large data set to approve its accuracy, reliability, and validity.

\section{Acknowledgment}

This paper has been extracted from parts of Mr. Masud Karimpur M.Sc. thesis supported by the Research Council of Shiraz University of Medical Sciences (94-01-07-9766). The authors would like to thank Center for Development of Clinical Research of Nemazee Hospital and Dr. Nasrin Shokrpour for editorial assistance.

\section{Conflict of Interest}

None 


\section{References}

1. Laal M. Technology in medical science. ProcediaSocial and Behavioral Sciences. 2013;81:384-8.

2. O'Sullivan T, Studdert R, editors. Handheld medical devices negotiating for reconfigurable resources using agents. Computer-Based Medical Systems, 2005 Proceedings 18th IEEE Symposium on; 2005: IEEE-p.70-5. doi: 10.1109/cbms.2005.63.

3. Kay M, Santos J, Takane M. mHealth: New horizons for health through mobile technologies. World Health Organization. 2011;64:66-71.

4. Meneghello J, Lee K, Gilleade K, editors. Mobile distributed processing of physiological data. Networked Embedded Systems for Every Application (NESEA), 2012 IEEE 3rd International Conference on; 2012: IEEE.p.1-8. doi: 10.1109/ nesea.2012.6474031.

5. Adibi S. Mobile health: a technology road map. New York: Springer; 2015.

6. K TH, A BB, Garan H, Sciacca RR, Riga T, Warren $\mathrm{K}$, et al. Evaluating the Utility of mHealth ECG Heart Monitoring for the Detection and Management of Atrial Fibrillation in Clinical Practice. J Atr Fibrillation. 2017;9:1546. doi: 10.4022/jafib.1546. PubMed PMID: 29250277; PubMed Central PMCID: PMC5673393.

7. Secerbegovic A, Mujčić A, Suljanović N, Nurkic M, Tasic J, editors. The research mHealth platform for ECG monitoring. Telecommunications (ConTEL), Proceedings of the 2011 11th International Conference on; 2011: IEEE.p.103-8.

8. Stalberg E, Falck B. The role of electromyography in neurology. Electroencephalogr Clin Neurophysiol. 1997;103:579-98.doi: 10.1016/s00134694(97)00138-7.PubMed PMID: 9546485.

9. Brannagan TH, Hays AP, Lange DJ, Trojaborg W. The role of quantitative electromyography in inclusion body myositis. J Neurol Neurosurg Psychiatry. 1997;63:776-9.doi: 10.1136/jnnp.63.6.776. PubMed PMID: 9416815; PubMed Central PMCID: PMC2169851.

10. Fuglsang-Frederiksen A. The role of different EMG methods in evaluating myopathy. Clin Neurophysiol. 2006;117:1173-89. doi: 10.1016/j. clinph.2005.12.018. PubMed PMID: 16516549.

11. Farkas C, Hamilton-Wright A, Parsaei $H$, Stashuk DW. A review of clinical quantitative electromyography. Crit Rev Biomed Eng. 2010;38:467-85. doi: 10.1615/critrevbiomedeng.v38.i5.30.PubMed PMID: 21175409.

12. Hodges PW, Bui BH. A comparison of computerbased methods for the determination of onset of muscle contraction using electromyography. Electroencephalogr Clin Neurophysiol. 1996;101:5119.doi: 10.1016/s0013-4694(96)95190-5.PubMed PMID: 9020824.

13. Oh SJ. Clinical electromyography: nerve conduction studies. Philadelphia: Lippincott Williams \& Wilkins; 2003.

14. Kimura J. Electrodiagnosis in diseases of nerve and muscle. Oxford: Oxford University Press; 2013.

15. Nikolic M, Krarup C. EMGTools, an adaptive and versatile tool for detailed EMG analysis. IEEE Trans Biomed Eng. 2011;58:2707-18. doi: 10.1109/ TBME.2010.2064773. PubMed PMID: 20699205.

16. Stalberg E, Falck B, Sonoo M, Stalberg S, Astrom M. Multi-MUP EMG analysis--a two year experience in daily clinical work. Electroencephalogr Clin Neurophysiol. 1995;97:145-54.doi: 10.1016/0924980x(95)00007-8.PubMed PMID: 7607102.

17. Farina $D$, Fattorini L, Felici F, Filligoi G. Nonlinear surface EMG analysis to detect changes of motor unit conduction velocity and synchronization. J Appl Physiol (1985). 2002;93:1753-63. doi: 10.1152/japplphysiol.00314.2002. PubMed PMID: 12381763.

18. Tomberg C, Levarlet-Joye H, Desmedt JE. Reaction times recording methods: reliability and EMG analysis of patterns of motor commands. Electroencephalogr Clin Neurophysiol. 1991;81:269-78. doi: $\quad 10.1016 / 0168-5597(91) 90013-n . P u b M e d$ PMID: 1714821.

19. Di Fabio RP. Reliability of computerized surface electromyography for determining the onset of muscle activity. Phys Ther. 1987;67:43-8.doi: doi. org/10.1093/ptj/67.1. PubMed PMID: 3797476.

20. Parsaei H, Stashuk DW, Rasheed S, Farkas C, Hamilton-Wright A. Intramuscular EMG signal decomposition. Crit Rev Biomed Eng. 2010;38:435-65. doi: $\quad 10.1615 /$ critrevbiomedeng.v38.i5.2.PubMed PMID: 21175408.

21. Parsaei H, Stashuk DW. EMG signal decomposition using motor unit potential train validity. IEEE Trans Neural Syst Rehabil Eng. 2013;21:265-74. doi: 10.1109/TNSRE.2012.2218287. PubMed PMID: 23033332.

22. Ozgunen KT, Celik U, Kurdak SS. Determination of an Optimal Threshold Value for Muscle Activity Detection in EMG Analysis. J Sports Sci Med. 2010;9:620-8. PubMed PMID: 24149789; PubMed Central PMCID: PMC3761824.

23. Holla S, Katti MM. Android based mobile application development and its security. International Journal of Computer Trends and Technology. 
2012;3:486-90.

24. Henriques $G$, Lamanna L, Kotowski $D$, Hlomani $H$, Stacey $D$, Baker $P$, et al. An ontology-driven approach to mobile data collection applications for the healthcare industry. Network Modeling Analysis in Health Informatics and Bioinformatics. 2013;2:213-23.

25. Lee Y, Ashton-Miller JA. Age and gender effects on the proximal propagation of an impulsive force along the adult human upper extremity. Ann Biomed Eng. 2014;42:25-35. doi: 10.1007/ s10439-013-0900-9. PubMed PMID: 23979475; PubMed Central PMCID: PMC3872510.

26. Pressman RS, Maxim B. Software engineering: a practitioner's approach. 8th ed. New York: Mc-
Graw-Hill Education; 2014.

27. Jinjin $\mathrm{H}$, Zhaolin F. The design of ERP in the Multitier architecture. Digital Manufacturing and Automation (ICDMA), 2013 Fourth International Conference on. 2013:1441-4.

28. Alam MM, Hati S, De D, Chattopadhyay S, editors. Secure sharing of mobile device data using public cloud. Confluence The Next Generation Information Technology Summit (Confluence), 2014 5th International Conference-; 2014: IEEE.p.149-54. 10.1109/confluence.2014.6949344.

29. Bhati S, Sharma S, Singh K. Review On Google Android a Mobile Platform. IOSR Journal of Computer Engineering (IOSR-JCE) e-ISSN. 2013:22780661. 